\title{
Visual Communication and the Map: How Maps as Visual Objects Convey Meaning in Specific Contexts
}

\begin{abstract}
Amy Propen
This article reports the results of a case study of two maps, produced by the National Marine Fisheries Service and the Natural Resources Defense Council, and their involvement in a federal court case over the deployment of the Navy's low-frequency active sonar. Borrowing from Kress and van Leeuwen's (1996) approach to visual analysis, Turnbull's (1989) understanding of the map, and Latour's (1990) understanding of how visuals work in social contexts, the article offers an analytical approach to studying maps as powerful visual, rhetorical objects.
\end{abstract}

On August 7, 2002, the Natural Resources Defense Council (NRDC) filed a lawsuit in a San Francisco federal court against the Navy and National Marine Fisheries Service (NMFS), requesting a preliminary injunction to "halt Navy deployment of SURTASS LFA sonar" in areas of the North Pacific Ocean (Calvert \& Buck, 2005, p. 3). Low-frequency active sonar (LFA) is used to detect submarines but has also shown to be extremely harmful to marine mammals. In October 2002, the court granted the preliminary injunction, and in August 2003, a federal court issued a permanent injunction restricting deployment of the LFA sonar and ordering the Navy to negotiate with plaintiffs (the NRDC) the terms of its deployment. In October 2003 the Navy reached a settlement with the NRDC, agreeing to limit LFA deployment particularly in areas of the North Pacific Ocean. In August 2002, when the initial lawsuit was filed, a map produced by the NRDC helped persuade the court to grant the October 2002 hearing for preliminary injunction. During that preliminary injunction hearing, the NRDC map was invoked in debates over whether the NMFS would deploy LFA sonar in areas small enough to meet "the requirement of a "specific geographical region," as set forth in the Marine Mammal Protection Act (MMPA; NRDC v. Evans, 2002 , p. 7). Briefly put, the MMPA has strict guidelines that set parameters on 
any human activity that may result in what the MMPA calls the "taking" (harming, harassing, or killing) of marine mammals; because the deployment of LFA sonar could result in the taking of marine mammals, the NMFS had to show that any incidental takings would occur within the parameters specified by the MMPA's requirements for specified geographical region.

In this hearing, the NRDC map was discussed in relation to a map produced by the NMFS, which also made claims about the specified geographical region affected by the sonar. According to Negin (2003), "during the trial, the National Marine Fisheries Service, which supported the Navy plan, produced a map that supposedly showed where the sonar would be deployed" (p. 46), in essence defining the areas in which marine mammals would be exposed to its effects. This map made the area of affected ocean appear much smaller than it actually was, whereas the NRDC map of the same area of ocean more accurately portrayed the (much larger) area involved. The NRDC map "helped persuade the court to grant the preliminary hearing," which then took place in October 2002 (Luijten, personal communication, 2004). Together, these maps served as the basis for a debate about where the sonar could be deployed, and who was representing the area more accurately.

Of course, additional variables beyond MMPA compliance were taken into consideration during the October $\mathbf{2 0 0 2}$ motion for preliminary injunction, such as the NMFS' alleged violation of the National Environmental Policy Act and the Endangered Species Act. That is, visuals rarely work in isolation from other texts and contexts, and the maps in this case functioned as one part of a larger debate over whether the Navy/NMFS violated several environmental laws through the use of LFA sonar. However, the role of these two maps in the discussion of whether the NMFS violated the MMPA by misrepresenting the specified geographical region in which the sonar was to be deployed is the main focus of this study of how maps function as persuasive, visual, rhetorical artifacts.

This small case study analyzes the work of these visuals in and around a rhetorical situation. First, the study looks at how the map, as a visual diagram, participates in meaning making. That is, the Navy/NMFS and the NRDC could have used only the numerical, longitudinal, and latitudinal coordinates to describe where the LFA sonar would be deployed; however, they chose to visually represent the affected area. How, then, does the map make meaning as a visual representation? Here, a visual analysis of the maps that draws from the theories of Turnbull (1989) and Kress and van Leeuwen (1996) provides a helpful framework for describing how maps make meaning in social contexts. The two maps are compared to show how each sends subtly different messages about the geographic area involved, and consequently, the number and species of marine mammals per region affected by the sonar. To describe how each map shapes and communicates a different message, of course, pertains to how the map works in social contexts-an idea that Latour's (1990) work helps to illuminate. Accordingly, this analysis emphasizes the map's 
communicative and persuasive power relative to issues such as its audience, purpose, and context.

\section{ON SELECTION OF THE CASE AND DATA COLLECTION}

I first learned of this case from the Summer 2003 issue of Onearth-a periodical published by the NRDC. After reading Negin's (2003) article, "Battle Maps: In the War of Words, Sometimes a Picture Can Win the Day," I contacted Joep Luijten, then the NRDC's Geographic Information Systems (GIS) manager. At that time, he was kind enough to share with me the map he produced for the NRDC in reference to this case, as well as direct me to the .PDF document (available online to the public) that contained the NMFS map to which the NRDC responded. Though Luijten was the GIS specialist who originally created the map in Figure 2, the map belongs to the NRDC, and so I refer to it as such. Between 2003 and 2005, I kept in e-mail contact with Luijten regarding questions and clarifications about the case. I also received permission from the NRDC to reprint their map in this article (and since it was used in open court, it is not considered confidential). The map produced by the NMFS is available to the public on the Internet. I am indebted to Luijten not only for sharing the NRDC map with me in the first place, but also for his invaluable feedback throughout my research.

The analysis offered in this study has certain limitations that should be acknowledged. First, although I have confirmation that the NRDC map was presented at the October 2002 preliminary injunction hearing, I have not been able to clarify exactly when the NMFS map was submitted to the court, and whether it was used in an oral argument or submitted to the court as an attachment with other documents. That is, although the NRDC and NMFS maps were involved in the case, I cannot speak to the precise setting in which they were invoked and discussed, largely because I have been unable to attain the actual court transcripts for the case. What I have been able to acquire, however, is the NRDC attorneys' brief for the initial lawsuit filed in August 2002, the Opinion of the Court for the preliminary injunction hearing (October 2002), and the Opinion of the Court for the permanent injunction summary (August 2003). Most important to this analysis is the Opinion of the Court for the preliminary injunction hearing. It is here that the NRDC map is invoked and referred to as Exhibit A, and it is here that the MMPA's requirement of "specified geographical region" is discussed in relation to the NRDC map and to allegations that the NMFS was in violation of these requirements. Although the NMFS map is not directly mentioned in the Opinion of the Court for the preliminary injunction hearing, the citation at the bottom of the NRDC"s "Authorized Deployment" map (Figure 2) confirms that their map is indeed based on the NMFS' (as I note later on), which helps to convey that the two maps are in dialogue with 
one another and make competing claims to knowledge. ${ }^{\prime}$ It is these competing knowledge claims that I am most interested in, and subsequently, how the maps function in the rhetorical situation. However, before exploring these issues, it is first necessary to understand how the map itself functions as a visual object.

\section{CONVENTIONS AND FEATURES OF THE MAP}

Previous work has understood the map as bearing communicative, and in many cases, persuasive power. In their landmark essay, "Ideology and the Map: Toward a Postmodern Visual Design Practice," for example, Barton and Barton (1993) spoke to this point well, and in many ways, this article builds on some of the terrain initially covered by them. That is, they understand the map as an ideologically charged, cultural artifact and advocate a "critical study of the ideology of visuals" (p. 50). They suggest that visual representations-maps in particular-are "seen as complicit with social-control mechanisms inextricably linked to power and authority" (p. 53). They understood maps as integral to the "conduct of war" and, quoting from the well-known cartographer Denis Wood, noted that the "implicit agenda" and "real uses" of most maps "are to possess and to claim, to legitimate and to name" (p. 54). Maps, Barton and Barton tell us, should be understood as legitimating certain interests while deligitimating and marginalizing other interests. As a case in point, they cite the geographic distortions perpetuated by the Mercator projection of the world map (wherein the visual rendering of Russia is twice the size of Africa), noting that the Mercator projection contributes to the visual distortions that "are embodied in the cartographic space as a grid," (p. 58) and thus the grid functions as an ideologically charged representational device that distorts while its goal is to convey accurate models of the terrain by positioning space along equal lines of latitude and longitude.

Important to note for the purposes of this article, however, is that Barton and Barton's (1993) cultural analysis of mapping practices is for them one piece of a larger puzzle, the purpose of which is to engage the question of what constitutes good visual design; as such, their discussion ultimately returns to the domain of visual design practice-specifically, the "proposal of a more inclusionary visual design practice" (one which they do well to describe, and one which I myself advo-

\footnotetext{
'Because the Opinion of the Court does not refer to the National Marine Fisheries Service (NMFS) map by name, however. I cannot state with absolute certainty that the Court ever specifically referred to the map in Figure I during the preliminary injunction hearing. However, based on my conversations with the Natural Resources Defense Council (NRDC), the similarity between the NMFS map and the surrounding discussions in the Opinion of the Court, and the fact that the NRDC map is based on the NMFS map in Figure 1, I feel there is a high degree of likelihood that the NMFS map was at least implicitly involved during the preliminary injunction hearing.
} 
cate; p. 76). In other words, their work uses the map as a means by which to focus on a more progressive notion of visual design practice, whereas I am more interested in the map itself as a rhetorical object that has the capacity to describe contested space and make what sometimes turn out to be competing claims to knowledge. This small case study, then, is not only compatible with and builds on much of what Barton and Barton described, but also extends their landmark work by holding a lens to the notion that maps can be communicative and persuasive objects. By looking to Turnbull (1989) and especially to Latour (1990), their discussion can be extended by examining how maps can work as power levers and tap into existing social contexts to convey meaning and contribute to the rhetorical situation.

Maps can be also understood as visual artifacts that tap into and rely on a visual culture, which Henderson (1995) defined as "a way of seeing that reflects and contributes to the specific manner in which one renders the world," or "a particular way of seeing the world that is linked to explicit material experience" (pp. 197-198). Maps not only reflect renderings of the world, but they also create these renderings. This creation process relies on particular cartographic conventions that shape how the map makes meaning.

Maps are useful devices of visual communication because they provide the nonexpert with a particular perspective about a place; that is, the map can help paint a picture about where things are and how the landscape ought to be perceived. Bourdieu (1999) described the role of the map in this regard. The map, he said, "is the analogy which occurs to an outsider who has to find his way around in a foreign landscape and who compensates for his lack of practical mastery...by the use of a model of all possible routes" (p. 2). Although Bourdieu spoke of a highway or road map, one that provides multiple options for getting from one place to another, the issue of audience raised by his definition is pertinent to this study. That is, the map is a helpful, supplemental device to nonexperts when they must make decisions that require spatial understanding. And, as discussed later, experts produce maps that represent the landscape in specific ways when they need to describe to nonexperts specific issues or problems related to that place, or when they need to propose problem solutions to decision makers. What this means is that the map is selective. Its creators make choices about what to include and exclude based on what they know about the problem at hand, their understanding of social contexts, and their knowledge of audience - all of which are effective practices in technical communication.

Turnbull (1989) elucidated the definition of the map, the design conventions particular to the map, and how such definitions and conventions are inherently context-bound. He asked: "What are maps and what are their function? What is the difference between a map and a picture? What is the relation of the map to the landscape it represents? How do you 'read' a map?" (p. 1). He cited spatiality as "a central element in almost all our representations of the world" (p. 1). Although this 
study focuses on how maps function in Western culture, Turnbull rightly noted that, "while spatiality may indeed be fundamental to all cultures, what actually counts as the 'relative location' of particular objects may not be quite so basic and may constitute one of the variables that differentiate the way cultures experience the world" (p. 2). Particularly relevant is Turnbull's discussion of the dependence of Western cartographic practice on the use of the grid to help order and fix space: "Those who are imbued with what is sometimes called 'the Western world view" think of objects as having fixed characteristics and defined boundaries" (p. 3 ). Turnbull also pointed out that the grid "is a human construct," and that "there is nothing in reality that corresponds to [the] grid" (p. 26). Thus, a generic convention of the map in Western culture is its imposition of the grid onto the landscape it represents. The map also creates meaning through its selectivity, or the inclusion and exclusion of information; of course, the map cannot possibly account for or "display all there is to know about any given piece of the environment," but if a visual representation of space is to be deemed a map, Turnbull agreed that it "must directly represent at least some aspects of the landscape" (p. 3).

Borrowing from the semiotic theory of Peirce, Turnbull (1989) noted that cartographic representation generally falls into two main categories: iconic and symbolic. An iconic representation bears a direct likeness to the feature it describes; it attempts "to directly portray certain visual aspects of the piece of territory in question," (p. 3) whereas a symbolic representation relies on and taps into social or institutional contexts to make meaning; it makes use of "purely conventional signs and symbols, like letters, numbers, or graphic devices" (p. 3). Many Western maps employ both iconic and symbolic features. In doing so, maps do not necessarily distinguish between the two modes of representation; rather, these two modes are implicit in cartographic convention and representation.

Maps are thus context bound and create meaning through the use of particular cartographic conventions, such as the construct of the grid, the expectation that at least some aspects of the landscape are represented, and their use of both iconic and symbolic features. How, then, can maps be defined? Turnbull (1989) said:

Maps are graphic representations that facilitate a spatial understanding of things, concepts, conditions, processes, or events in the human world. ... [The map is a] graphic representation of the milieu, containing both pictorial (or iconic) and nonpictorial elements. Such representations may include anything from a few simple lines to highly complex and detailed diagrams. (p. 3)

To think about the purpose of the map and its ability to facilitate spatially based understanding also illuminates another key aspect of how maps make meaning, and that is its use of projection.

As Barton and Barton (1993) also noted, the round Earth cannot be projected onto a flat, two-dimensional surface without some level of distortion; as a result, 
"different modes of projection have been developed" to account for this issue (Turnbull, 1989, p. 6). Although "no one projection is the best or the most accurate," different types of projections have different purposes for which they are more or less well suited: "A particular projection is selected by the mapmaker on the basis of functional and perhaps aesthetic criteria, or because of a specification or convention" (p. 6). For example, the Mercator projection, which is one of the most widely used projections, and traditionally used in sea navigation, "has the characteristic that the distance between parallels (the horizontal lines at specific latitudes) increases," the farther away one moves from the equator (Luijten, personal communication, 2003). The Mercator projection has the effect of distorting relative size, most prominently toward the poles, though, and consequently, "the area of Greenland and Antarctica is shown proportionally (too) large" (Luijten, personal communication, 2003). However, because "the direction of any straight line drawn in this projection represents the actual compass bearing," the Mercator projection, although well suited for its use in sea navigation charts, would be inappropriately used in calculating area (Luijten, personal communication, 2003).

\section{THE MAP AS AN OBJECT OF VISUAL COMMUNICATION}

Based on this discussion of the conventions and features of the map, the map can be understood as an object of visual communication. To build a stronger case for such an understanding, though, it is also necessary to define what I mean by "visual communication"; however, to do so also requires that we define the terms visual literacy, visual communication, and visual rhetoric, because they are often used interchangeably. These three terms, however, actually build on one another, and should thus be understood differently. I look to the definition of visual literacy posited by the International Visual Literacy Association (IVLA). They define the term as:

A group of vision competencies a human being can develop by seeing and at the same time having and integrating other sensory experiences. The development of these competencies is fundamental to normal human learning. When developed, they enable a visually literate person to discriminate and interpret the visual actions, objects, and/or symbols, natural or man-made, that are [encountered] in [the] environment. Through the creative use of these competencies, [we are] able to communicate with others. Through the appreciative use of these competencies, [we are] able to comprehend and enjoy the masterworks of visual communications. (IVLA, 2002, p. 1)

Given the emphasis on how cognition and development pertain to learning and sensory experience (though we might complicate what counts as normal human 
learning), visual literacy falls more so in the provenance of studies in human cognition and perception. Dondis's (1986) theory of visual literacy, which relies heavily on Gestalt psychology, is also concerned with cognition and perception. Dondis saw visual literacy as a practice or a methodology "that makes it possible to educate all people to their maximum ability both as makers and receivers of visual messages" (p. 1). Although the analytical approach I am advocating is less concerned with studies in cognition and perception, what the IVLA's definition affords is a view of the map as a visual object, symbol, or masterwork of visual communication (IVLA, 2002). Visual literacy is also of concern to the mapmaker, who must have the capacity to be selective in making choices about what is to be included and excluded in the map. Thus, it follows that to be visually literate is to be able to create objects that communicate visually, or to be able to participate in visual communication. We might then understand visual literacy as logically prior to visual communication.

In this article, I start from the point of the mapmaker already being visually literate (though versions of visual literacy may of course vary given the context or knowledge of the rhetorical situation) and I work from there to analyze and critique the product of the cartographer's craft-the object of visual communication, or the map itself. Visual communication thus refers in this article to any visual artifact that takes into account, implicitly or explicitly, the communicative principles specific to the genre of that particular artifact. For example, the map, as a visual object, counts as a map because it takes into account particular cartographic conventions given its purpose, audience, and context. Because communication is often the territory for rhetoric, what follows from an understanding of the map as a device of visual communication is the fact that the map often, if not always, conveys a particular point of view: "In one sense, maps display information. They tell us where things are and how to get there. But a map also visually constructs a way of understanding a place and thus makes a deliberative argument" (Faigley, George, Palchik, \& Selfe, 2004, p. 388).

Given a view of rhetoric that takes the map as a visual artifact whose epistemic circumstance is dependent on both cartographic convention and social contexts, it becomes necessary to account for an understanding of rhetoric that moves beyond its traditional purview, and also accounts for the complexities of analyzing visual artifacts in specific contexts. I thus subscribe to an understanding of visual rhetoric expressed in a position statement prepared by Blakesley, in response to a question posed at the 2003 Alliance of Rhetoric Societies conference on the status and future of rhetorical studies. When asked the question, "What is visual rhetoric, and what is its tradition," Blakesley (2003) replied:

To understand visual rhetoric better, we need to reanimate its tradition, and in doing so, reconsider our conception of rhetoric itself as primarily a verbal art. The differentiation of the verbal and visual runs deep not only in rhetoric but in mainstream epistemology. I propose that, rather than perpetuate this division 
of the verbal and visual, we now need to consider their common basis in perception. What we find is that even in everyday verbal expression (orally or in writing) there are profoundly complex visual components. We find also that there are verbal (and rhetorical) components in everyday acts of seeing. What insights does our rhetorical tradition provide on the nature of visual rhetoric? On the nature of seeing and its relationship to verbal and rhetorical processes? Is there a visual component in identification? Analogy? Metaphor? The bending of the will? What is the tradition of visual rhetoric, and how will it change our understanding of the rhetorical tradition(s)? (p. 1)

Here, Blakesley (2003) called for a visual rhetoric that starts not from the assumption of a visual/verbal divide, but rather from an assumption of the potentially symbiotic relation between the verbal and visual. Blakesley's call to reconceive the traditional view of rhetoric as primarily a verbal art resonates well with Wood and Fels's (1986) notion of the map as comprised of both word and image: "As word lends icon access to the semantic field of its culture, icon invites word to realize its expressive potentials in the visual field. The result is the dual signification virtually synonymous with maps, and the complementary exchange of meaning that it engenders" (p. 80). Table 1 summarizes the distinctions between visual literacy, visual communication, and visual rhetoric as I understand them to be.

By thinking of maps not solely as objects of visual communication, but also as rhetorical objects, it becomes even easier to see how the meaning engendered in the following two examples eventually results in competing knowledge claims.

TABLE 1

Distinctions Between Visual Literacy, Visual Communication, and Visual Rhetoric

Eisual literacy
and sensory experience, or concerned largely with studies in
human cognition and perception.
To be visually literate is to be able to discriminate and interpret
visual actions and objects in the world, and eventually to be able
to create objects that communicate visually; ultimately, visual
literacy enables participation in the act of visual communication.
We may therefore understand visual literacy as logically or
conceptually prior to visual communication.
Refers to any visual artifact that takes into account, implicitly or
explicitly, the communicative principles specific to the genre of
that particular artifact.
Builds on the idea that communication is often the territory for
rhetoric, that not all visual objects communicate neutral,
objective information; rather, many, if not most, visual objects
are interested or ideologically charged. Objects of visual
communication may therefore also be rhetorical.




\section{A VISUAL ANALYSIS OF THE MAPS PRODUCED BY THE NMFS AND NRDC}

The map in Figure 1, titled: "MMPA [Marine Mammal Protection Act] Small Take Authorization Determinations: 1) Will incidental takings occur in a specified geographical area? Yes." was produced in 2002 by the NMFS and used by the Navy (National Oceanic and Atmospheric Association Fisheries, 2002).

This map is part of a larger Microsoft PowerPoint ${ }^{\circledR}$ presentation involving the Navy's request to deploy LFA sonar in areas of the North Pacific Ocean. The MMPA defines "take" as: "to harass, hunt, capture, collect, or kill, or attempt to harass, hunt, capture, collect or kill, any marine mammal" (NRDC v. Evans, 2002, p. 6). To receive a "small take authorization," an activity must be restricted to a "spec-

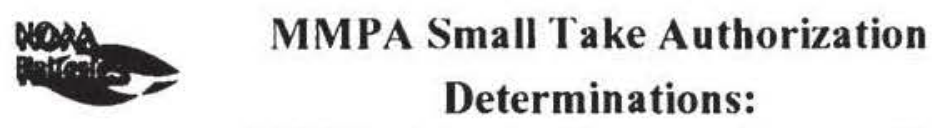

1) Will incidental takings oceur in a specified

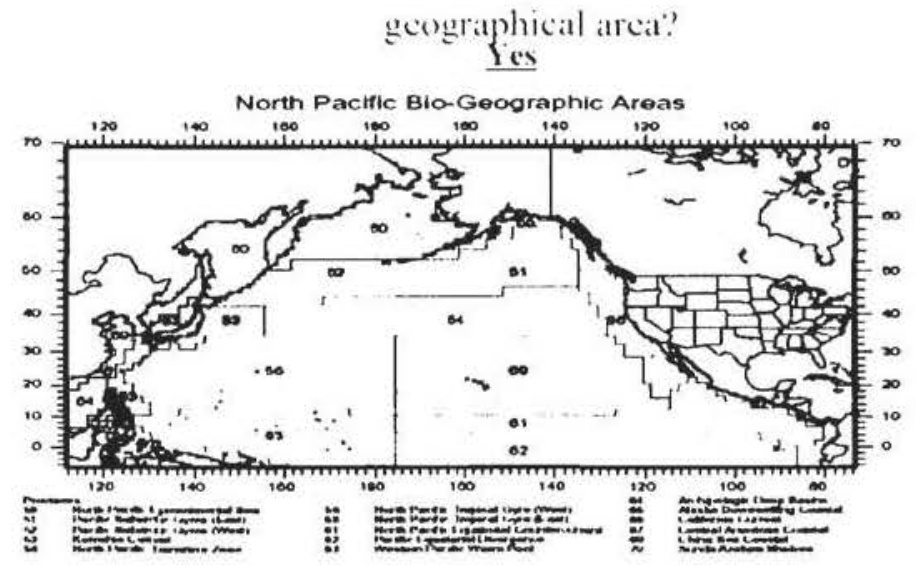

Inset from above:
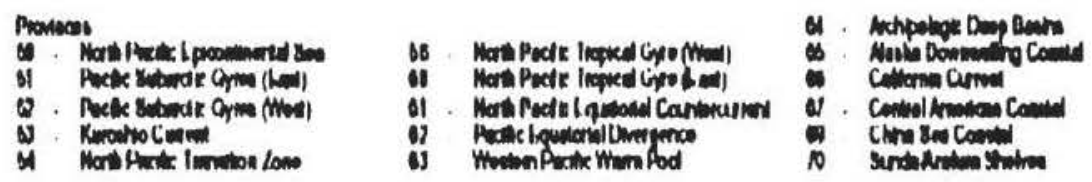

FIGURE 1 MMPA Small Take Authorization Determinations: 1) Will incidental takings occur in a specified geographical area? Yes. 
ified geographical region," resulting in the taking of a small number of marine mammals, and have only a "negligible impact" on the species (NRDC v. Evans, 2002 , p. 6). Small take authorizations are issued by the Secretary, who must then "provide for the monitoring and reporting of such takings" (p. 6). Further, "specified geographical region" is defined by the Federal Code of Regulations as "an area within which a specified activity is conducted and which has similar biogeographic characteristics" (NRDC v. Evans, 2002, p. 8). Such a region needs to be "narrowly identified so that the anticipated effects will be substantially similar" (p. 8). That is, it would be "inappropriate to identify the entire Pacific Coast of the North American continent as a specified geographical region, but it may be appropriate to identify particular segments of that coast having similar characteristics, both biological and otherwise, as specified geographical regions" (p. 8).

Turnbull (1989) might find the map in Figure 1 to be both iconic and symbolic. It is iconic because it bears a likeness to the physical territory it represents- the North Pacific Ocean and its surrounding landforms. The North Pacific Ocean is centered in the map, making it the focus of the visual, whereas the surrounding coast is present to provide perspective. The map is symbolic because it makes use of conventional symbols such as lines and numbers and graphical devices such as the use of color. The ocean is divided into what are called "provinces," or "biogeographic areas." Each area is referred to by its name and a number between 50 and 70, and is also assigned a color. For example, Province 56, pictured at the center of the map in light blue, is called the North Pacific Tropical Gyres West. Province 60, to the right of Province 56 and shown in light purple, is the North Pacific Tropical Gyres East. Visually, it appears from this map that these are the two largest provinces to be affected by deployment of the LFA sonar, although it is difficult to make this assumption or assertion based solely on looking at the NMFS map; this is because nowhere in the NMFS map does it suggest that LFA sonar is actually deployed in Provinces 56 or 60, and nowhere in this map is the viewer provided with the actual area of each province. The use of color in the NMFS map can also be read as problematic. That is, one might assume upon first reading that the color used in the NMFS map might indicate which areas of ocean will be affected by LFA sonar. However, there is no legend in the map and no discussion in its surrounding presentation that clearly indicates the use of color to convey particular information about any of the provinces; that is, why one province is blue, another green, or another purple. These choices do not seem to be explained anywhere. However, Provinces 53 and 67, both coastal, are indicated in a pink/red color. The Navy claims that only limited areas of ocean are affected by the LFA sonar, which would seem to be consistent with highlighting in red the smaller, coastal Provinces 53 and 67. Further, Tufte (1983) suggested that red is often used to indicate a higher level of importance than other colors: "Color often generates graphical puzzles. Despite our experiences with the spectrum in science textbooks and rainbows, the mind's eye does not readily give a visual ordering to colors, except possibly for red to reflect higher levels than other colors" (p. 154). However, because 
there is no legend or indication of the size of each province, it is not possible to know for certain what the color of these areas refers to, and thus it is not possible to make any connection between color and deployment of the LFA sonar.

Turnbull (1989) and Kress and van Leeuwen (1996) might also agree that these maps are selective in the information they represent. That is, these areas are part of an analytical process that "relates participants in terms of a part-whole structure" (Kress \& van Leeuwen, 1996, p. 89). This process "involves two kinds of participants: one Carrier (the whole) and any number of Possessive Attributes (the parts)" (p. 89). In this map, for example, the North Pacific Ocean is the carrier, and each province is a possessive attribute that functions within the carrier. Further, Turnbull and Kress and van Leeuwen would agree that the choice of which attributes are made salient within the carrier is a selective process:

Maps may provide quite distinct analyses of what seems to be the same Carrier. Some maps focus on geographical features such as waterways, altitude, etc.; others concentrate on social and political boundaries. Analysis always involves selection. Some attributes or characteristics of the Carrier are singled out as criterial in the given context, or generally, while others are ignored, treated as nonessential and irrelevant. (Kress \& van Leeuwen, 1996, p. 90)

Thus, given the purpose for which these maps are used, the selection of attributes within the carrier has to do with the fact that the NMFS wants to depict which provinces will be affected by deployment of the LFA sonar. It is necessary, then, that their map of the North Pacific Ocean should focus on making these provinces visible while ignoring other potential possessive attributes within the carrier. Such choices inevitably draw the viewer's attention to the implicated provinces, not only because the provinces are called out through their use of color even if the colors used are not intuitive, and are referred to by name, but also because other aspects of the background, such as the surrounding coast and landforms, are muted or only lightly drawn to provide context. In other words, the map conveys meaning through the analytical processes invoked and manifested in its visual representation. To present the clearest meaning, however, the map must convey an understanding of its purpose and context. There are some interpretive problems with the NMFS map that manifest when viewing it both in the context of its intended use and in comparison with the NRDC's version. The map produced by the NRDC (Figure 2) in response to the NMFS map, on the other hand, seems to display a clearer understanding of context and purpose.

The map in Figure 2 was produced by the NRDC; as noted at the bottom of the map, the location of biogeographic regions in the NRDC map is based on that of the NMFS representation: 

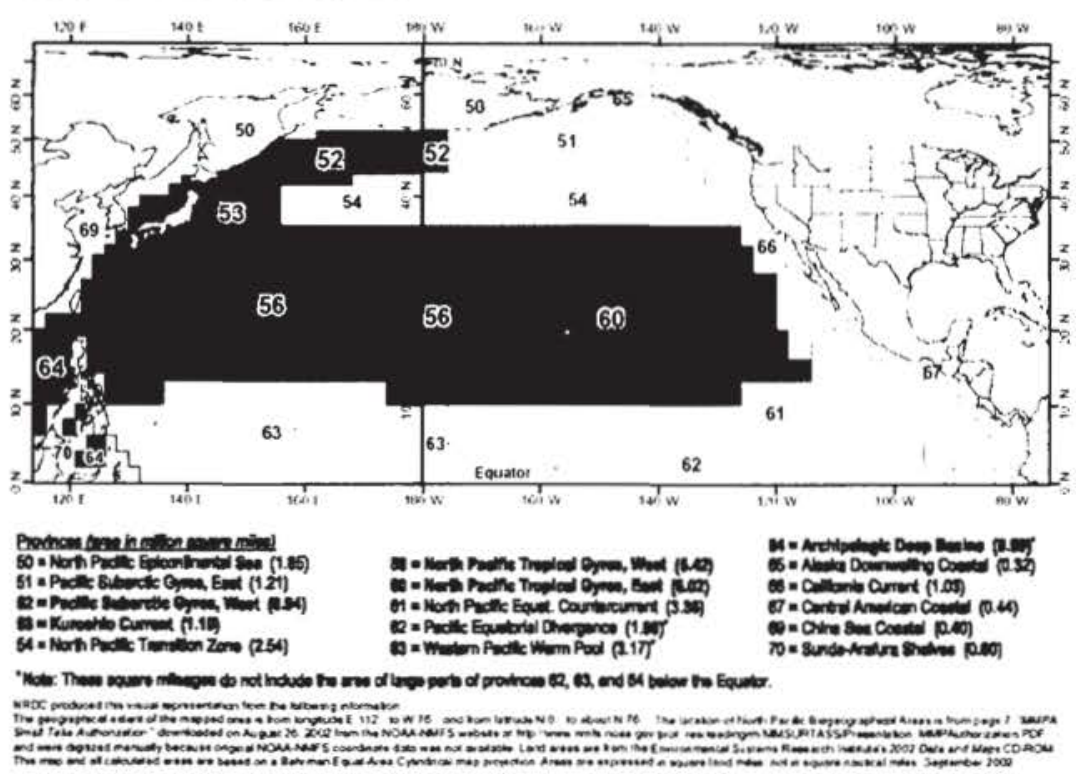

Inset from above:

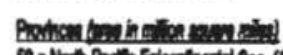

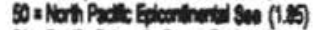

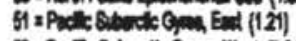

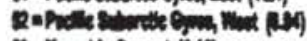

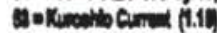

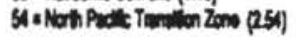

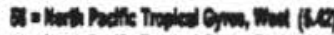

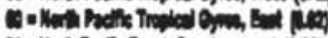

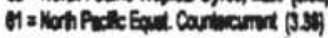

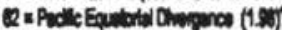

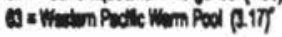

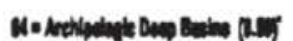

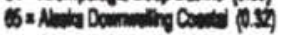

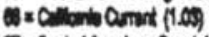

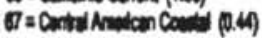

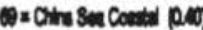

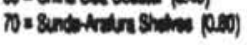

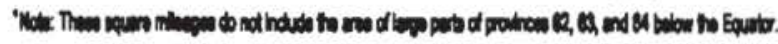

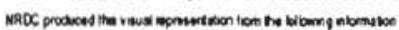

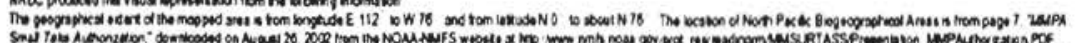

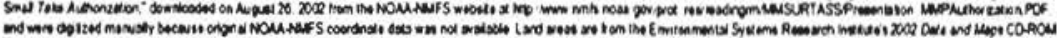

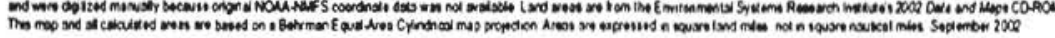

FIGURE 2 Authorized Deployment of LFA: 2002-2003. (NRDC).

NRDC produced this visual representation from the following information:

The geographical extent of the mapped area is from longitude $\mathrm{E} 112^{\circ}$ to $\mathrm{W}$ $76^{\circ}$ and from latitude $\mathrm{N} 0^{\circ}$ to about $\mathrm{N} 76^{\circ}$. The location of North Pacific Biogeographical Areas is from page 7, 'MMPA Small Take Authorization,' downloaded on August 26, 2002, from the NOAA-NMFS website at http:// www.nmfs.noaa.gov/prot_res/readingrm/MMSURTASS/Presentation_ 
MMPAuthorization.PDF, and were digitized manually because original NOAA-NMFS coordinate data were not available. Land areas are from the Environmental Systems Research Institute's 2002 Data and Maps CD-ROM. (NRDC, 2002)

There are several important differences between the two maps. Most apparent is the NRDC's clearer use of color. Possessive attributes within the carrier are highlighted in bright red and a legend indicates that areas highlighted in red correspond with areas of "LFA-affected Ocean." Although the NRDC's use of red may simply reflect their in-house style guide for cartographic convention, the color red, as Tufte (1983) suggested, may also be viewed as symbolic of warning in Western culture; it is therefore possible, perhaps, to understand the LFA-affected Ocean as dangerous. According to geographer Denis Wood (1992), the legend functions as a sign system:

The role of the legend is less to elucidate the "meaning" of this or that map element than to function as a sign in its own right ... the legend refers not to the map (or at least not directly to the map), but back, through a judicious selection of map elements, to that to which the map image itself refers. (p. 101)

In other words, Wood (1992) said that the legend itself is a sign that refers back to the idea of LFA-affected ocean in some capacity. The legend in the NRDC map does not visually represent the biogeographic regions in physical, geographical terms, as the map itself does; rather, it represents criterial aspects of the object, the LFA-affected ocean.

Recall also that it is not possible to discern from the NMFS map the actual size of each biogeographic region in the North Pacific Ocean. The NRDC map, on the other hand, provides its viewer with the actual area, in million square miles, for each province. For example, the NRDC map shows Province 56 to be 5.42 million square miles, and Province 60 to be 6.02 million square miles. This information, coupled with the information conveyed by the legend, allows the reader to see that provinces 56 and 60 are the largest areas to be affected by the LFA sonar. The fact that it is not possible to discern from the NMFS map area in square miles seems problematic, given the purpose for which the map was used. That is, the Navy's claim was that the LFA sonar would affect only a specified, contained geographical area of the North Pacific Ocean; however, the NMFS map is based on the Mercator projection, which makes it unsuitable for calculating area.

The Mercator projection has the effect of distorting relative size, most prominently toward the poles, and consequently, "the area of Greenland and Antarctica is shown proportionally (too) large" (Luijten, personal communication, 2003). However, because "the direction of any straight line drawn in this projection represents the actual compass bearing," the Mercator projection, although well suited for its use in sea navigation charts, would be inappropriately used in calculating 


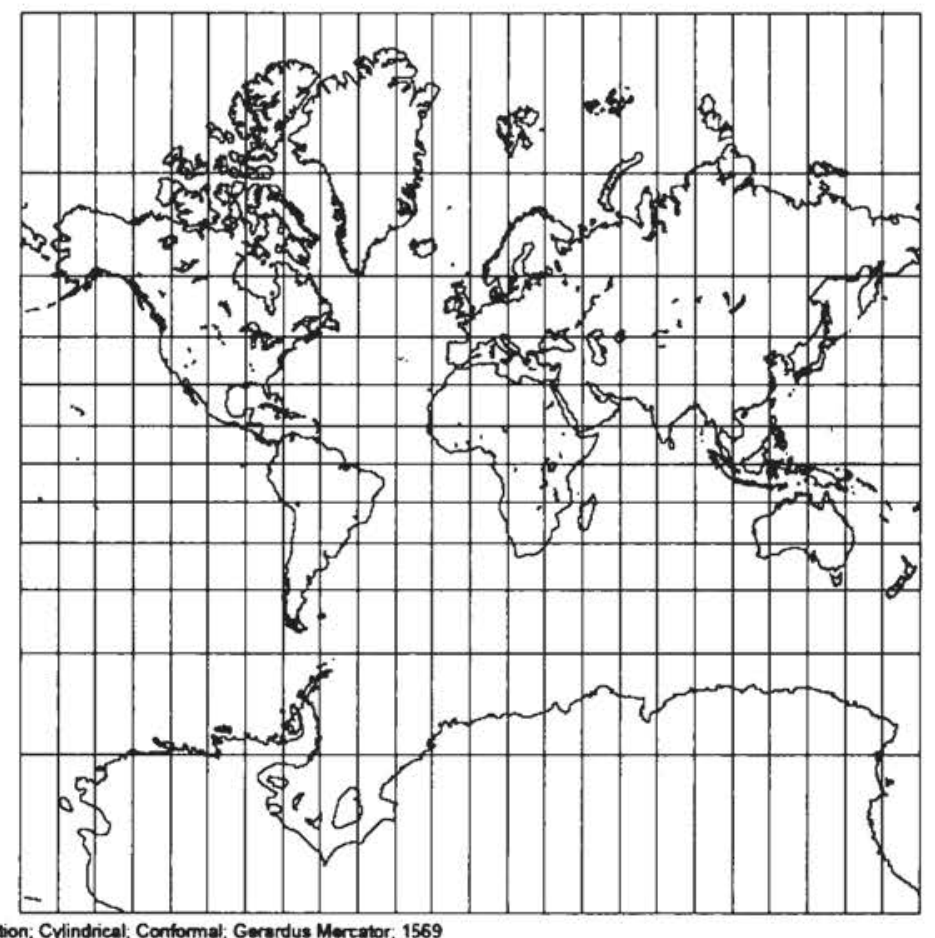

FIGURE 3 Sample Mercator projection (Anderson, 2004b).

area (Luijten, personal communication, 2003). The NRDC, on the other hand, understood that one purpose of the map was to describe the area of ocean affected by the LFA sonar, and therefore based their map on the Behrman Equal-Area projection, which is "in many ways the opposite of the Mercator projection: (1) distances between parallels decrease toward the poles; (2) the ... projection is not suitable to measure compass bearing; and (3) areas are maintained properly anywhere on the globe" (Luijten, personal communication, 2003).

Looking at the NRDC map, it is possible to see that the distance between parallels indeed decreases toward the poles; it is visually apparent that the area of the North pole appears compressed in this map, as a result of the use of the Behrman projection. The map in Figure 1, based on the Mercator projection, appears the opposite; the area toward the North pole appears taller than, or at least as tall as the area of ocean shown in the rest of the map. Although the NRDC could have countered the NMFS map by making their own Mercator-based map that only revised aspects such as the inclusion of a legend, or the use of color, they recognized that the choice of projection is a critical component of how maps make meaning, and as such, they would be remiss in neglecting to use a different projection in revising 


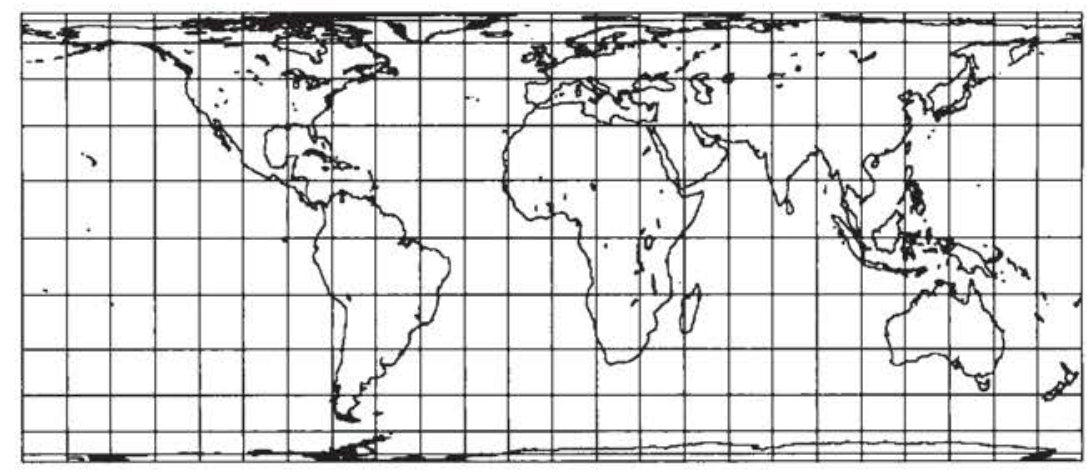

Behrmam propecton (Modfied Cyindncal Equat-area); Standard Parallolo = 30 Deg. NS. Wather Behrmann; 1910

FIGURE 4 Sample Behrman Equal-Area projection (Anderson, 2004a).

the NMFS map. I would extend this point to say that every visual medium (advertising image, scientific illustration, etc.) has its own set of critical design conventions that contribute to its ability to make meaning; when it comes to cartographic representation, the use of projection, although not the only convention specific to mapping, is one of the most critical. Thus, the NRDC map differs markedly from the NMFS map in terms of allowing for much clearer interpretation, and also in terms of more suitable use of projection, given the context and purpose of the map. It is more than just the appropriate choice of projection that gives the NRDC map its clarity, however; I suggest that it was the way in which the visual functioned as a whole, in terms of its clear use of color, and the inclusion of clearly described graphical features such as a legend, that gave the map its persuasive power and ultimately earned it recognition in the federal case between the Navy and the NRDC.

At the same time, to suggest that the NRDC map saved the day, so to speak, is to paint a naïve and overly simplistic portrait of how these maps functioned in the rhetorical situation. That is, as Latour (1990) saw it, no single visual can carry all the explanatory burden, nor can it alone account for the outcome of a given situation. What matters, he suggested, is how the map helps to create allies that can alleviate a difficult situation or avoid a potentially unfavorable outcome:

Who will win an agonistic encounter between two authors and between them and all the others they need to build up a statement $S$ ? Answer: the one able to muster on the spot the largest number of well aligned and faithful allies. ... My contention is that 
writing and imaging cannot by themselves explain the changes in our scientific societies, except insofar as they help to make the agonistic situation more favorable. Thus it is not all the anthropology of writing, nor all the history of visualization, that interests us in this context. Rather, we should concentrate on those aspects that help in the mustering, the presentation, the increase, the effective alignment, or ensuring the fidelity of new allies. (p. 24)

I agree with Latour's (1990) understanding of the limited power that any one visual can have in the rhetorical situation. That is, the NRDC map cannot by itself explain the outcome of the preliminary injunction hearing. As I mentioned at the outset of this article, the NRDC map functioned as one part of a larger courtroom debate over whether the NMFS violated several environmental laws through it deployment of LFA sonar.

\section{LIMITATIONS OF THE VISUAL AND THE LARGER CONTEXT}

The main purpose of the NRDC map was to point out the misrepresentation of area of LFA-affected ocean in the NMFS map, and with their map they were able to do so. However, there exist two main problems in the case of cartographic representation of specified biogeographic region: First, the number of million square miles affected by the sonar needs to be represented accurately. The NRDC was able to correct the NMFS's apparent error in portraying the area of affected ocean. Second, however, the map needs to be able to account specifically for LFA impact on marine mammal populations. That is, the NRDC is able to account for one issue by choosing a different map projection, but they still cannot explicitly account for the second problem, which is the problem of how to represent specified biogeographic region specifically with regard to marine mammal populations. The Opinion of the Court helps describe this issue. At the time of the preliminary injunction hearing, the biogeographic regions delineated by the NMFS

divid[ed] the oceans into 15 biomes, and 54 provinces within those biomes, as designed by Longhurst (1998).... NMFS stated that it believed that this approach met the statutory definition [of specified geographic region] because "a biome is the most likely geographic region to contain the majority of a specific marine mammal stock, especially those that are migratory." While admittedly, the Longhurst schematic was designed for plankton, it is the best scientific application available for designating specified geographic regions because no biogeographic concept has been designed for marine mammals. (NRDC v. Evans, 2002, p. 8)

Again, I cannot say with certainty that the "schematic" mentioned in the aforementioned quotation refers directly to the map in Figure 1; however, the map in Figure 
1 does also represent the North Pacific Ocean as containing 15 primary biomes, or biogeographic areas, which is generally consistent with the ongoing debate about the large size of the biogeographic areas affected by the sonar.

Surprisingly, the NMFS admits that their schematic was not designed specifically with marine mammals in mind, though they feel that their method is the best available option for designating specified geographic regions relative to marine mammal populations because no such concept exists for marine mammals. During the preliminary injunction hearing, the NRDC invited Rodney M. Fujita, a marine ecology specialist, to counter the argument that the NMFS schematic is appropriate for designating specified geographic regions relative to marine mammal populations. He stated that:

the Longhurst biomes are not particularly useful for estimating biological impacts on specific populations of marine mammals or other organisms. According to Fuijta, the provinces identified by NMFS are so large that each one contains many diverse habitats, species assemblages, and levels of productivity. ... "Even if NMFS' purpose in creating very large biogeographical provinces was to ensure that they contain whole stocks of migratory marine mammals, the boundaries are somewhat biologically arbitrary, failing to correspond to population distributions of gray whales, blue whales, and other species." (NRDC v. Evans, 2002. pp. 9-10)

Not only are the specified geographic areas larger than the NMFS represents them to be, but also, according to Fujita, these areas are too large to measure impacts on specific marine mammal populations.

During the preliminary injunction hearing, the NRDC objected to the biogeographic regions delineated by the NMFS. According to the court's opinion, the plaintiffs (NRDC)

object that the biomes and provinces identified by NMFS are still far too large. Plaintiffs have provided a map, attached as Exhibit $A$ to their motion, showing the very large size of some of these provinces. According to plaintiffs, Province 60 is larger than the continental United States and encompasses six million square miles of open ocean. The Court notes that Province 66 covers the entire Pacific coast from roughly Cabo San Lucas at the southern tip of Baja California to the Canadian border. Plaintiffs argue that if "it would be inappropriate to identify the entire Pacific coast of the North American Continent as a specified geographical region," ... then surely an area twice the size of the United States violates the MMPA. (NRDC v. Evans, 2002, p. 9. emphasis added)

The NRDC introduced their map (Figure 2) as Exhibit A in the preliminary injunction hearing to point out the large size of the provinces affected by LFA sonar. However, it is important to note that although the map indeed plays a significant role in shaping the court's perception of what counts as an accurately portrayed 
biogeographic region, other factors play a role as well. The NMFS claimed to use the representation they did because no alternative biogeographical scheme exists in which the specified biogeographic region also defines an area within which specific marine mammal populations reside. On one hand, they may be right, but on the other hand, the NRDC, using a more appropriate map projection, was at least able to make a more accurate assessment of the number of square miles comprised by each specified biogeographical region. By more accurately portraying the size of the affected areas, the assumption is that the areas will therefore more accurately represent marine mammal populations within each region. Because there is no definition of a "specified geographic region" specific to marine mammal populations, however, the NRDC is unable to show this correlation directly. Even though the NRDC is better able to represent the area of LFA-affected ocean than the NMFS, the broader implication of their map (that this representation will subsequently allow for accurate measurement of impact on marine mammals) is difficult to make explicit. Thus, the Opinion of the Court states:

Plaintiffs [the NRDC] have established serious issues with respect to whether NMFS violated the MMPA by choosing such undifferentiated geographical areas, particularly in light of the failure to carve out sufficient areas of special biological importance for feeding, breeding, and the like that lie within these large areas and make them less homogenous. ... Plaintiffs have not presented any evidence, however, disputing [the] NMFS' conclusion that no alternative biogeographical scheme currently exists for marine mammals that can readily be applied here. ... Although the NMFS' choices may be flawed, on this record they do not appear to be so flawed that the Court will likely invalidate them as arbitrary and capricious. At most, plaintiffs have raised a serious question on the merits. (NRDC v. Evans, 2002, p. 11)

Ultimately, the NRDC was successful in presenting a road block in the NMFS's attempt to deploy LFA sonar. That is, the Court eventually ruled:

Plaintiffs have shown that they are likely to prevail on establishing violations of the MMPA, NEPA [National Environmental Policy Act], ESA [Endangered Species Act], and the APA [Administrative Procedure Act]. ... Plaintiffs have also shown the possibility of irreparable harm to the marine environment that supports the existence of these species. (NRDC v. Evans, 2002, p. 37)

Accordingly, the Court granted the preliminary injunction, which resulted in the restriction of the sonar's use in particular areas of the North Pacific Ocean, and ordered both parties "to meet and confer on the precise terms of a preliminary injunction consistent with this opinion" (NRDC v. Evans, 2002, p. 39). In the interim, the Court stated that "defendants should not deploy LFA sonar" (p. 39). Following this case, in August 2003, a permanent injunction was issued, which restricted the Navy's use of LFA sonar. Although the Court agreed that the NRDC raised serious 
questions with regard to whether the NMFS violated the criteria for specified geographic region, as set forth in the MMPA, it did not feel that the NRDC presented a solution to the entire problem. That is, the NMFS may have misrepresented the area of affected ocean; however, because there seems to be no precedent for a schematic that not only accurately portrays the area, but also accounts for the marine mammal populations therein, there are limits to just how much of the situation the NRDC map can account for. It is at this point that we see not only the merits of the map as persuasive, visual representation, but its limits as well.

\section{CLOSING THOUGHTS}

As Turnbull (1989) noted when he quoted from Latour, for Latour, power is "the consequence of association," of the ability of words and images to make connections that mobilize and "muster allies on the spot-allies, that is, in the struggle over what is to count as a fact" (Turnbull, 1989, p. 55). The claims made by the NRDC and NMFS, regarding the definition of "specified geographic region" are indeed claims rooted in the struggle over what gets to count as a fact. Because the NRDC map enabled them to "establish serious issues" regarding whether the NMFS violated the MMPA "by choosing such undifferentiated geographical areas," they were able to muster allies in the struggle over what gets to count as a fact (NRDC v. Evans, 2002, p. 11). Even though their map was limited in its ability to explicitly show evidence of an "alternative biogeographical scheme" that could be applied for marine mammals, the map was still able to "raise serious questions on the merits" of the NMFS' claims (p. 11). Thus, the NRDC was able to gain an ally in the Court, at least in terms of the Court's agreement that the NMFS likely misrepresented the specified geographic areas involved in LFA deployment.

Notable too is the fact that the NRDC map was introduced in the court case as "Exhibit A." and presented to the Court as evidence. Does a visual presented as evidence differ from a visual that is persuasive for potentially other reasons? Of course, it is tricky to assume that anything submitted as evidence automatically counts as persuasive, visual or not; rather. I think the assumption, or at least the expectation therein, is that anything submitted as evidence will likely count as persuasive. Although it is beyond the scope of this study to address such questions in depth, I suggest for now that the reference to the map as Exhibit $A$ may have certainly afforded it a certain credibility, but it was not this credibility that made the map effective.

Rather, one lesson to be gleaned from this small case study is that maps function as visual, rhetorical objects. Although they rarely work alone, and usually function with or within other texts and contexts, they are nonetheless powerful knowledge-making devices. As we have seen in the case of the NRDC and NMFS, when cartographic representation adequately accounts for audience, purpose, and con- 
text, it can successfully illuminate issues and relations that other mediums cannot. Conversely, this case also reveals the limitations of the work that one visual can accomplish. An additional point needs to be made here regarding the map's relevance to technical and scientific communication. Luijten, the GIS specialist who originally created the map for the NRDC, thought that the visual medium in and of itself, as opposed to written text, made a difference in the case: "Luijten emphasizes that it's not so much the information but the medium that makes his work powerful. 'In the real world, you have only a couple of minutes to get your point across to decision makers.' ... 'In those cases, a map can be more helpful than a 30-page document'” (Negin, 2003, p. 46). Finally, I would extend Luijten's point to say that, in addition to the efficiency and appropriateness of the visual medium itself, in this case, the clarity with which the biogeographic regions were portrayed, the choice of map projection given the context, and the clear use of conventional signs and symbols all greatly contributed to the NRDC map's ability to play a role in environmental decision making that ultimately resulted in the protection of marine mammal populations.

\section{ACKNOWLEDGMENTS}

I am grateful to the invaluable feedback of the anonymous peer reviewers and to Joep Luijten for his input during the writing and revision process.

\section{REFERENCES}

Anderson, P. B. (2004a). Cylindrical map projections: Behrman projection. Map Projections. Retrieved January 7, 2005, from http://www.ilstu.edu/microcam/map_projections/Cylindrical.html/

Anderson, P. B. (2004b). Cylindrical map projections: Mercator projection. Map Projections. Retrieved January 7, 2005, from http://www.ilstu.edu/microcam/map_projections/Cylindrical.html/

Barton, B. F., \& Barton, M. S. (1993). Ideology and the map: Toward a postmodern design practice. In N. Roundy \& C. Thralls (Eds.). Professional communication: The social perspective (pp. 49-78). Newbury Park, CA: Sage.

Blakesley, D. (2003). What is visual rhetoric, and what is its tradition? Retrieved September, 2005 from http://www.comm.umn.edu/ARS/Tradition/blakesley,\%20tradition.htm/

Bourdieu, P. (1999). Outline of a theory of practice (R. Nice, Trans.). Cambridge, England: Cambridge University Press.

Calvert, K., \& Buck, E. H. (2005). Active sonar and marine mammals: Chronology with references. Congressional Research Service: The Library of Congress. Retrieved October, 2005, from http:// www.fas.org/sgp/crs/weapons/RS22158.pdf/

Dondis, D. A. (1986). A primer of visual literacy. Cambridge, MA: MIT Press.

Faigley, L., George, D., Palchik, A., \& Selfe, C. (2004). Picturing texts. New York: Norton.

Henderson, K. (1995). The visual culture of engineers. In S. L. Star (Ed.), The cultures of computing (pp. 196-217). Oxford, England: Blackwell. 
International Visual Literacy Association. (2002). What is visual literacy? Retrieved September, 2005. from http://www.ivla.org/organization/whatis.htm/

Kress, G., \& van Leeuwen. T. (1996). Reading images: The grammar of visual design. New York: Routledge.

Latour, B. (1990). Drawing things together. In M. Lynch \& S. Woolgar (Eds.), Representation in scientific practice (pp. 19-68). Cambridge, MA: MIT Press.

National Oceanic and Atmospheric Association Fisheries. (2002). MMPA small take authorization determinations: 1) Will incidental takings occur in a specified geographical area? Yes. MMPA Small Take Authorization, 7. Retrieved September, 2006, from http:/www.nmfs.noaa.gov/pr/pdfs/permits/ mmpa_small_take.pdf\#search=\%22MMPA \%20small\%20take\%20authorizations\%22

Natural Resources Defense Council v. Evans, United States District Court for the Northern District of California. (2002). Opinion of the court: Opinion and order granting plaintiffs' motion for a preliminary injunction. Docket number C-02-3805 EDL.232 F. Supp.2d 1003 (N.D. Cal. 2002).

Natural Resources Defense Council. (2002). Authorized deployment of LFA: 2002-2003 [Map.] Washington, DC: Natural Resources Defense Council.

Negin, E. (2003). Battle maps: In the war of words, sometimes a picture can win the day. Onearth, 25. 46.

Tufte, E. (1983). The visual display of quantitative information. Cheshire, CT: Graphics Press.

Turnbull, D. (1989). Maps are territories, science is an atlas: A portfolio of exhibits. Chicago: University of Chicago Press.

Wood. D. (1992). The power of maps. New York: Guilford.

Wood, D., \& Fels, J. (1986). Designs on signs: Myth and meaning in maps. Cartographica, 23, 54-103.

Amy Propen is a doctoral candidate in the Department of Rhetoric and Technical and Scientific Communication at the University of Minnesota. Her research interests include visual communication, semiotics, rhetorical theory, cultural studies, and mapping. 